OPEN

SUBJECT AREAS:

CARDIOVASCULAR

DISEASES

ENZYMES

Received

28 January 2014

Accepted

11 August 2014

Published

9 October 2014

Correspondence and requests for materials should be addressed to L.M.B. (I.m.brewster@ amc.nl)

\title{
Creatine kinase inhibits ADP-induced platelet aggregation
}

\author{
D. L. Horjus' ${ }^{1}$, R. Nieuwland ${ }^{2}$, K. B. Boateng' ${ }^{1}$, M. C. L. Schaap ${ }^{2}$, G. A. van Montfrans ${ }^{1,3}$, J. F. Clark ${ }^{5}$, A. Sturk ${ }^{2}$ \\ \& L. M. Brewster ${ }^{1,3,4}$
}

'Dept. of Vascular Medicine, Academic Medical Center, University of Amsterdam, Amsterdam, the Netherlands, ${ }^{2}$ Dept. of Laboratory Medicine, Academic Medical Center, University of Amsterdam, Amsterdam, the Netherlands, ${ }^{3}$ Dept. of Internal Medicine, Academic Medical Center, University of Amsterdam, Amsterdam, the Netherlands, ${ }^{4}$ Dept. of Social Medicine, Academic Medical Center, University of Amsterdam, Amsterdam, the Netherlands, ${ }^{5}$ Dept. of Neurology, Cincinnati Children's Hospital, Cincinnati, Ohio, USA.

Bleeding risk with antiplatelet therapy is an increasing clinical challenge. However, the inter-individual variation in this risk is poorly understood. We assessed whether the level of plasma creatine kinase, the enzyme that utilizes ADP and phosphocreatine to rapidly regenerate ATP, may modulate bleeding risk through a dose-dependent inhibition of ADP-induced platelet activation. Exogenous creatine kinase (500 to $4000 \mathrm{IU} / \mathrm{L}$, phosphocreatine $5 \mathrm{mM}$ ) added to human plasma induced a dose-dependent reduction to complete inhibition of ADP-induced platelet aggregation. Accordingly, endogenous plasma creatine kinase, studied in 9 healthy men (mean age 27.9 y, SE 3.3; creatine kinase 115 to 859 IU/L, median 358), was associated with reduced ADP-induced platelet aggregation (Spearman's rank correlation coefficient, -0.6; $p<0.05)$. After exercise, at an endogenous creatine kinase level of 4664, ADP-induced platelet aggregation was undetectable, normalizing after rest, with a concomitant reduction of creatine kinase to normal values. Thus, creatine kinase reduces ADP-induced platelet activation. This may promote bleeding, in particular when patients use platelet $\mathrm{P} 2 \mathrm{Y}_{12}$ ADP receptor inhibitors.

leeding risk associated with antiplatelet therapy for prevention or treatment of cardiovascular events is an increasing clinical challenge, in particular in the context of multiple drug therapy ${ }^{1-4}$. There is a strong association between bleeding and adverse outcomes ${ }^{1,2}$. In patients with acute coronary syndromes enrolled in 3 large studies, OASIS, OASIS-2, and CURE, major bleeding events resulted in a 5-fold higher incidence of death during the first 30 days, and a 1.5 fold higher incidence of death between 30 days and 6 months ${ }^{1}$. International societies hasten up development of management strategies to minimize bleeding and subsequent adverse consequences in acute thrombolysis with coronary syndromes and percutaneous coronary intervention, while reports on greater bleeding risk with platelet P2 $\mathrm{Y}_{12}$ ADP receptor inhibitors raise concern ${ }^{1-4}$. However, it remains unclear, why some patients have greater bleeding risk than others ${ }^{1-4}$.

We hypothesize that inter-individual differences in plasma creatine kinase (CK, EC 2.7.3.2) activity modulates bleeding risk. The enzyme utilizes ADP to catalyze the reversible transfer of a phosphoryl group (P) from phosphocreatine, yielding ATP and creatine ${ }^{5-7}$ :

$$
\mathrm{ADP}+\text { Pcreatine }+\mathrm{H}^{+} \leftrightarrow \mathrm{ATP}+\text { Creatine }
$$

High CK activity commonly occurs after exercise and in patients with damage of CK-rich tissue, including cardiac muscle, brain, and skeletal muscle ${ }^{5,6,8}$. Moreover, the inter-individual variation in CK activity in the general population is wider than previously suggested, ranging from below 25 to up to $5000 \mathrm{IU} / \mathrm{L}^{5}$, with particularly high levels in men and in persons of African ancestry. Taking the central role of ADP in platelet activation into account, we assessed whether plasma CK activity inhibits ADP-induced platelet aggregation.

\section{Methods}

Study design and ethics statement. The protocol was approved by the Medical Ethical Committee of our hospital, and all participants gave their written informed consent before enrolment. The methods were carried out in accordance with the approved guidelines. In this singlecenter study, we executed a 3-step conditional protocol. In the first step, the main CK isoenzyme in human blood, CK MM was added in a clinically relevant activity range ( 500 to $4000 \mathrm{IU} / \mathrm{L}$ ) to low-CK plasma obtained from women ${ }^{5}$, to assess the effect on platelet aggregation. The target CK activity levels for the second and third step of the protocol depended on the outcome of the first step. In the second step, we planned 
to assess platelet aggregation in healthy men with relatively low to high endogenous $\mathrm{CK}$ activity levels. Finally, we assessed platelet aggregation with highly elevated CK after exercise.

Study population. All participants were volunteers recruited by public advertisements. We included healthy adults, aged $<50$ years, BMI $\leq 30 \mathrm{~kg} / \mathrm{m}^{2}$, who did not smoke, used no prescription or other drugs, and had no personal or family history of bleeding or muscle disease, and no personal history of sickle cell disease, diabetes mellitus, or other disorder. Furthermore, physical examination and laboratory studies needed to be without abnormalities. Extended laboratory studies were performed after overnight fasting in the participants of step 2 of the protocol, including haemoglobin, sickle cell test (by haemoglobin phenotyping), thrombocyte count, lipid spectrum (total and HDL cholesterol; triglycerides), thyroid stimulating hormone (since subclinical hypothyroidism may increase plasma CK) ${ }^{8}$, ASAT, ALAT, alkaline phosphatase, gamma-GT, albumin, renal function (creatinine, eGFR), coagulation tests (activated partial thromboplastine time and prothrombine time), and intrinsic platelet function (as assessed by platelet aggregation in platelet rich plasma upon addition of ADP 1,2 and $5 \mu \mathrm{M}$; collagen $1 \mu \mathrm{g} / \mathrm{mL}$; arachidonic acid 1 and $2 \mathrm{mM}$; ristocetin 1.25 and $2.5 \mathrm{mg} / \mathrm{mL}$ ).

CK and platelet aggregation. Platelet aggregation was assessed in by light transmittance aggregometry (PAP-8E platelet aggregation profiler, Bio/Data Corporation, Horsham, PA, USA). Citrate-anticoagulated blood $(0.32 \%)$ was centrifuged (Rotina 420R, Hettich Lab Technology, Tuttlingen, Germany) during 15 minutes at $180 \times \mathrm{g}$ to obtain platelet rich plasma. Platelet poor plasma was prepared by 10 minutes centrifugation at $1550 \times$ g. Experiments were performed at $37^{\circ} \mathrm{C}$ under stirring conditions. Thrombin receptor-activated peptide (TRAP; $15 \mu \mathrm{M}$, Bachem, Bubendorf, Switzerland) was used to induce maximum platelet aggregation as an indicator to interpret the ADP aggregation curves. In the first step of the protocol, the addition of external CK, ADP-induced platelet aggregation was assessed at the lowest ADP concentration $(1 \mu \mathrm{M})$ where a second aggregation wave was visible, in the presence of both phosphocreatine (CrP; $5 \mathrm{mM}$, Sigma-Aldrich, St. Louis, MO, USA) and human CK MM isoenzyme (Sigma-Aldrich), at 500, 1000, 2000, and $4000 \mathrm{IU} / \mathrm{L}$ At the threshold ADP concentration the aggregation is likely to be very sensitive to the CK activity, but differs between subjects and could therefore not be chosen for the second and third part of the studies. In the second step, the assessment of endogenous $\mathrm{CK}, \mathrm{ADP}$-induced platelet aggregation was performed at ADP $2 \mu \mathrm{M}$ before and after supplementation with CrP $5 \mathrm{mM}$, both in excess. In the third step, 1 day after intensive exercise, ADP-induced platelet aggregation was assessed at ADP $(2 \mu \mathrm{M})$ without other additions. In all steps of the protocol, the main readout parameter was the area under the aggregation curve after 10 minutes.

Statistical analysis. The main outcome was the association between endogenous CK activity and ADP-induced platelet aggregation. Based on reported differences in ADP-induced platelet aggregation in subgroups of the population known to have high vs. low circulating CK activity, we conservatively estimated to find a negative correlation between endogenous CK activity and platelet aggregation, with a Spearman's correlation coefficient of $-0.6^{5,9}$, and calculated we needed at least 7 participants for this outcome, to assess this difference with an alpha of 0.05 and a 1 - beta of 0.80 .

The correlation between the area under the curve of the platelet aggregation test after 10 minutes and endogenous plasma CK activity was calculated using Spearman's rank correlations coefficient. Since the direction of the outcome was postulated before the experiment, a one-tailed $p$ value of 0.05 or less was considered statistically significant, to avoid or reduce bias in favor of $\mathrm{H}_{0}$. Data are expressed as mean $+/-\mathrm{SE}$, unless stated otherwise. Data were analyzed using SPSS Statistics version 22 for Windows (IBM SPSS, New York, NY, USA).

\section{Results}

All eligible healthy volunteers met the inclusion criteria. In the first experimental step, we added exogenous CK (500 to $4000 \mathrm{IU} / \mathrm{L}$ ) to plasma obtained from 7 healthy white women (mean age $23.1 \mathrm{y}, \mathrm{SE}$ 0.8 ; mean BMI $21.6 \mathrm{~kg} / \mathrm{m}^{2}$, SE 0.7 ) with relatively low endogenous plasma CK activity ( 45 to $128 \mathrm{IU} / \mathrm{L}$, median 82 ). With higher doses exogenous $\mathrm{CK}, \mathrm{ADP}$-induced platelet aggregation became increasingly attenuated, and was completely abolished at CK $4000 \mathrm{IU} / \mathrm{L}$ (a typical aggregometer tracing is depicted in Figure 1, Panel A).

Secondly, platelet aggregation was assessed with relatively low to high endogenous CK activity, in 9 healthy men, 4 of African ancestry; with a mean age of $28.8 \mathrm{y}$ (SE 3.3), and a mean BMI of $23.6 \mathrm{~kg} / \mathrm{m}^{2}(\mathrm{SE}$ 0.9). Plasma CK activity in these men ranged from 115 to 859 IU/L (median 358). Thrombocyte count was within the normal range (151 to $257 \times 10^{9} / \mathrm{L}$, median 203 ). Furthermore, we detected no intrinsic platelet disorders, and activated partial thromboplastin time or prothrombin time were within the normal range, with a mean duration of respectively 27.0 (SE 0.5) and 11.3 (SE 0.2) seconds. The remaining laboratory tests as described in the method section were without abnormalities. In the platelet aggregation assay, baseline ADP-stimulated area under the aggregation curve was 415.7 (SE 113.1). With ADP and creatine phosphate added, the area under the curve was reduced to 66.6 (SE 16.2), a difference of 349.1, with a $95 \%$ CI of (113.3 to 584.9); $p$ 0.005. Importantly, we found a greater reduction in area under the aggregation curve with higher endogenous CK activity, with a Spearman's rank correlation coefficient of $-0.59 ; p$ 0.046 , indicating that the ADP-induced platelet aggregation became increasingly inhibited at higher levels of CK.

Finally, as a proof of principle experiment, one of the male volunteers underwent intensive exercise to achieve a highly elevated plasma CK activity (4664 IU/L) after 1 day, without clinical signs of rhabdomyolysis. At this $\mathrm{CK}$ level, the ADP-induced platelet aggregation was completely absent, with a negligible area under the curve (Figure 1, Panel B). As expected, plasma CK activity returned to a normal level (258 IU/L) after 7 days of rest ${ }^{5,6}$, with a concomitant complete normalization of ADP-induced platelet aggregation.

\section{Discussion}

To our knowledge, this is the first report suggesting that plasma CK in clinical relevant ranges may reduce platelet aggregation. This finding may have clinical implications for patients and healthy subjects with high plasma CK activity, in particular when using antiplatelet or anticoagulant therapy.

$\mathrm{CK}$ is the main ADP scavenger within the circulation ${ }^{10}$. The enzyme can scavenge ADP as a binding protein, or convert ADP to ATP via its catalytic activity. The relative affinity of platelets and CK for ADP is approximately $10 \mu \mathrm{M}$. Traditional kinetic theories for the binding of a substrate (ADP) by competing binding sites (CK and platelets) often assume the substrate concentration to be infinitely high with a low concentration of binding sites. In the blood stream, this assumption is not valid. The platelet and CK concentration can change greatly compared to the ADP concentration and thereby become significant modulators of the free ADP concentration. Therefore, it is reasonable to suggest that when CK levels in the blood stream increase, with concomitant binding of its substrates via the random order $\mathrm{Bi}-\mathrm{Bi}$ ping-pong binding mechanisms, there will be a decrease in the concentration of free ADP available for activation of platelets.

In healthy people, intravascular CK is mainly the skeletal muscle type MM isoenzyme. This is thought to be due to a "proportional leak" from the abundant CK pool in healthy skeletal muscle, with the resulting level of plasma activity proportional to the level of intramuscular activity ${ }^{5,6}$. Several subgroups may have relatively high plasma CK levels. The highest intramuscular and plasma activities in healthy people are found in persons of sub-Saharan African ancestry. Furthermore, with exercise, increased lymphatic drainage is thought to lead to increased plasma CK activity within hours, and also damage of CK-rich tissue may lead to highly elevated plasma CK levels. In a random sample of the general population $(n=1444)$ we found values between 14 and 5783 IU/L (median 111) after 3 days of rest ${ }^{5}$.

These high CK levels may lead to less sticky platelets, and greater bleeding risk. In patients of African ancestry with ST-segment elevation myocardial infarction who underwent fibrinolysis, an unexplained higher risk of severe bleeding (adjusted OR 1.36; 95\% CI 1.14 to 1.62 ), with higher mortality (HR $2.83 ; 95 \%$ CI, 2.08 to 3.86 ) was found ${ }^{11}$, with similar findings in carotid endarterectomy ${ }^{12}$. Furthermore, patients of African descent displayed an unexplained greater risk of microbleeds and bloody stroke after adjustment for blood pressure (OR 3.31; 95\% CI, 1.14 to 9.57$)^{13}$. Notably, in the CHARISMA study, a high unexplained bleeding risk was found in the African ancestry subgroup with the use of the platelet ADP receptor inhibitor clopidogrel, (adjusted HR 3.78; 95\% CI, 1.35 to $10.60)^{14}$, despite lower plasma concentrations of the active drug ${ }^{15}$. Although these data support our hypothesis that high CK in this 


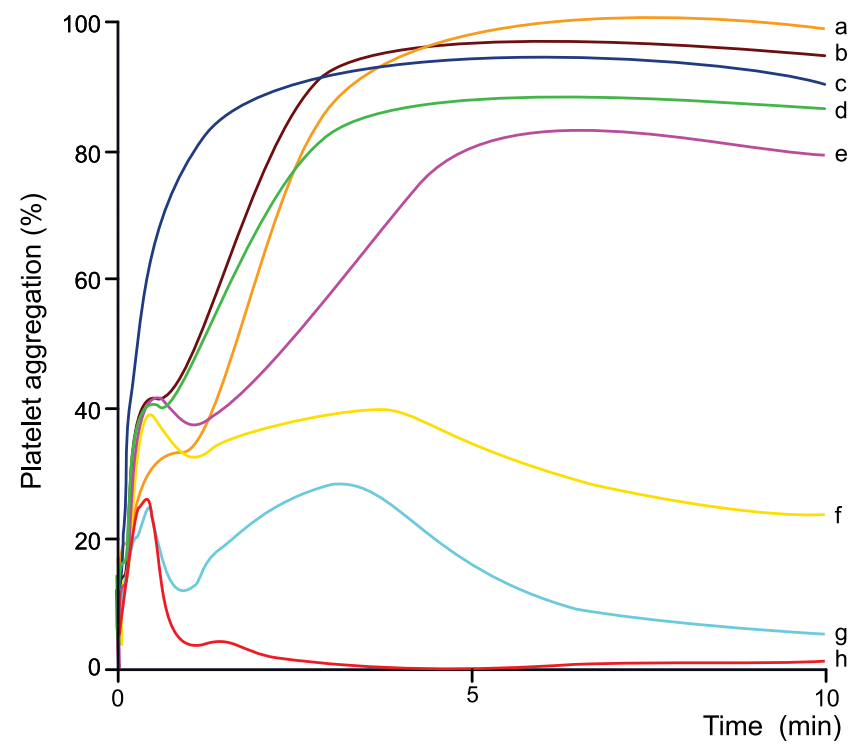

B

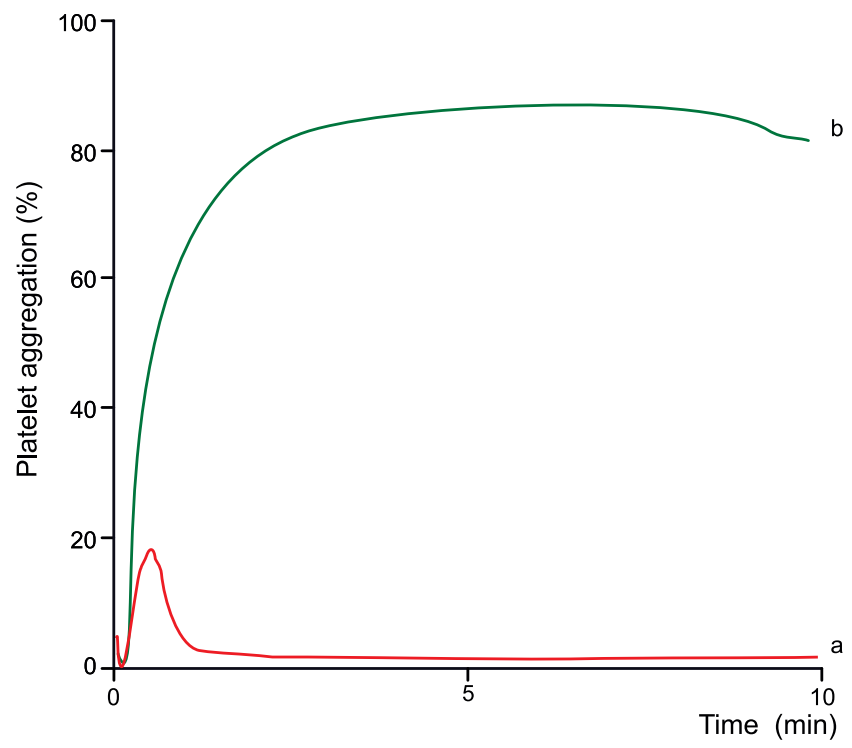

Figure 1 Panel a: Inhibition of ADP-induced platelet aggregation with increasing exogenous creatine kinase. Typical aggregometer tracing after exogenous creatine kinase, with tests performed at $1 \mu \mathrm{M}$ ADP. Platelet aggregation is depicted with (a) The substrates ADP and phosphocreatine (CrP; $5 \mathrm{mM}$ ), without addition of exogenous creatine kinase (CK) enzyme; (b) ADP and CK (4000 IU/L); (c) ADP and thrombin receptor activating peptide (TRAP; $15 \mu \mathrm{M}$ ); and (d) ADP only. Tests (e) to (h) were performed in the presence of both substrates, CrP and ADP. Importantly, with increasing exogenous CK enzyme added (respectively 500, 1000, 2000, and $4000 \mathrm{IU} / \mathrm{L}$ ), the second, irreversible phase of platelet aggregation became increasingly inhibited compared to the $100 \%$ aggregation reference with TRAP, to be completely abrogated at CK 4000 IU/L (h). Panel b: ADP-induced platelet aggregation with high endogenous creatine kinase after exercise. Tests were performed at $2 \mu \mathrm{M}$ ADP, without further addition. A day after a bout of intensive exercise, plasma creatine kinase (CK) activity was $4664 \mathrm{IU} / \mathrm{L}$, without signs of rhabdomyolysis. At this CK level, ADP-induced platelet aggregation was completely abrogated (a). After 7 days of rest, CK normalized to $258 \mathrm{IU} / \mathrm{L}$, with concomitant normalization of the ADP-induced platelet aggregation (b).

population subgroup may enhance bleeding risk, further clinical studies are warranted.

With regard to exercise-induced $\mathrm{CK}$ increase, acute strenuous exercise is reported to induce enhanced ADP-dependent platelet aggregation up during and up to one hour past the muscle strain, potentially related to increased sympathetic nervous system activity $^{16}$. However, lower ADP-induced platelet aggregability is generally found with moderate or regular exercise, or when aggregation is measured after a time lapse from hours to days after exercise $e^{17-19}$. The cause of the lower aggregability, linked to the health benefits of exercise, is not clear ${ }^{17}$, but the preliminary data presented in this paper indicate that the plasma creatine kinase ADP scavenging system, increasing in plasma activity within hours and peaking 1 to 2 days after exercise ${ }^{5,6}$, is a likely candidate to contribute to attenuated ADP-dependent platelet aggregation post-exercise.

Another subgroup of patients at risk of developing high plasma CK activity is statin users. Statins are associated with an increased hemorrhagic stroke risk, in particular in men. Although these drugs are reported to directly affect platelet function and coagulation in vitro, this does not explain clinical effects, and the true mechanism linking statins to brain haemorrhage remains a mystery ${ }^{20,21}$. Our observations on the potential role of CK might provide new clues, and even moderate elevations of plasma CK, as seen with statin use, might potentiate subclinical effects of statins on platelet function and increase bleeding risk.

Finally, after frank damage of CK-rich tissue such as striated muscle and brain tissue, plasma CK activities may be highly elevated, up to $>10,000 \mathrm{IU} / \mathrm{L}^{8,22-24}$. We propose that the unexplained uncontrollable haemorrhage reported in trauma victims and brain injury patients, a condition with a high mortality rate, might be due to high plasma CK.
In both "trauma-induced coagulopathy" and "traumatic brain injury platelet dysfunction", an unexplained near-complete inhibition of ADP-dependent platelet aggregation is observed ${ }^{25}$. Taking our data into account, highly elevated plasma CK known to occur after injury of CK-rich tissue $\mathrm{e}^{8,22-24}$ might contribute to the stark inhibition of ADP-dependent platelet aggregation in these conditions.

In the same line of thinking, this pathophysiology may include acute myocardial infarction. Although commonly associated with increased platelet reactivity, distinct hypoaggregability is noted up to 3 days after myocardial infarction, with hyperaggregability thereafter $^{26}$. We suggest that high CK levels appearing within a few hours after myocardial infarction, with a peak around 24 hours attenuating after 2 to 3 days $^{24}$, may be a factor that inhibits platelet aggregation. Since recent data suggest that low on-treatment platelet reactivity to $\mathrm{ADP}$ is associated with a higher risk of bleeding ${ }^{3}$, the use of (multiple) antiplatelet therapy might act synergistically in the individual patient with high CK to increase bleeding risk (Figure 2). Thus, the subgroups of patients with high CK may be particularly sensitive to the use of platelet ADP receptor blockers.

This study has strengths and limitations. We report herein an in vitro assay testing the effect of $\mathrm{CK}$ on human platelet aggregation, suggesting the possibility of coagulopathy with elevated plasma CK in the presence of phosphocreatine and ADP. The findings fit hitherto unexplained observations of reduced platelet aggregation or increased bleeding risk in population subgroups with a relatively high plasma CK such as statin users, and after exercise or tissue trauma. However, the sample size was rather small, and although evidence of statistical significance was found at only 9 participants, potentially indicating a major effect size of creatine kinase, the data should be considered hypothesis-generating, and larger studies will be needed to confirm our findings. Despite the strengths of this new 


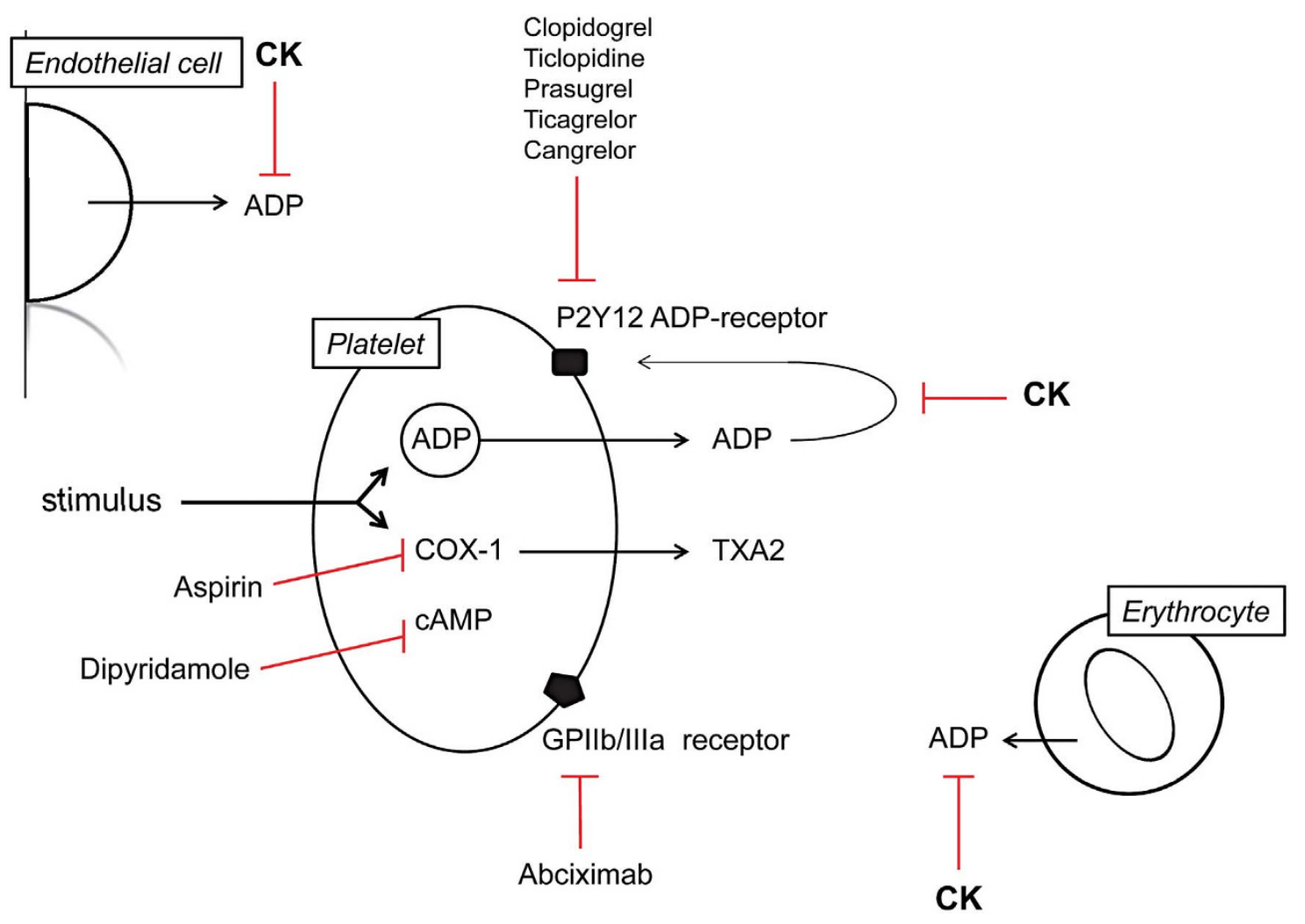

Figure $2 \mid$ Proposed mode of action of creatine kinase. Main inhibitory pathways of platelet activation and the potential role of plasma creatine kinase (CK) herein. COX-1, cyclooxygenase-1; TXA2, thromboxane A2. CK might attenuate platelet activation through scavenging plasma ADP as a binding protein, or via its catalytic activity convert ADP to ATP, leading to a reduced activation of the $\mathrm{P} 2 \mathrm{Y}_{12}$ ADP receptor and attenuated platelet aggregation.

concept, including biological plausibility, dose-dependency, and the existing trial evidence indicating that patients with high CK activity have a high bleeding risk, further clinical data are needed.

In summary, bleeding is an important complication of antiplatelet therapy, but gaps remain in knowledge of the underlying mechanisms ${ }^{1-4}$. To our knowledge, we are the first to present data indicating that high plasma $\mathrm{CK}$ in the range found within the general population, and in clinical conditions such as myocardial infarction, or damage of other CK-rich tissue such as skeletal muscle or brain may abrogate ADP-induced platelet activation. This may lead to higher bleeding risk in patients with high plasma $\mathrm{CK}$, in particular when on antiplatelet or anticoagulant therapy. Larger, prospective studies are needed to establish whether high plasma CK activity increases bleeding risk, and if so, the threshold CK activity at which this effect may occur, in particular with concomitant use of antithrombotic drugs.

1. Eikelboom, J. W. et al. Adverse impact of bleeding on prognosis in patients with acute coronary syndromes. Circulation. 114, 774-782 (2006).

2. Steg, P. G. et al. Bleeding in acute coronary syndromes and percutaneous coronary interventions: position paper by the Working Group on Thrombosis of the European Society of Cardiology. Eur Heart J. Doi:10.1093/eurheartj/ehr204 (2011)

3. Working Group on On-Treatment Platelet Reactivity. Tantry, U. S. et al. Consensus and update on the definition of on-treatment platelet reactivity to adenosine diphosphate associated with ischemia and bleeding. J Am Coll Cardiol. 62, 2261-73 (2013).

4. 2012 Writing Committee Members, Jneid, H. et al. American College of Cardiology Foundation; American Heart Association Task Force on Practice Guidelines. 2012 ACCF/AHA focused update of the guideline for the management of patients with unstable angina/Non-ST-elevation myocardial infarction (updating the 2007 guideline and replacing the 2011 focused update): a report of the American College of Cardiology Foundation/American Heart Association Task Force on practice guidelines. Circulation. 126, 875-910 (2012).

5. Brewster, L. M., Mairuhu, G., Sturk, A. \& van Montfrans, G. A. Distribution of creatine kinase in the general population: implications for statin therapy. Am Heart J. 154, 655-661 (2007).
6. Brewster, L. M. et al. Creatine kinase activity is associated with blood pressure. Circulation. 114, 2034-2039 (2006).

7. Djeza, P. P. \& Terzic, A. Phosphotransfer networks and cellular energetics. J Exp Biol. 206, 2039-2047. (2003).

8. Brewster, L. M. \& de Visser, M. The significance of increased creatine-kinase activity in the serum of patients without primary neuromuscular disease. Ned Tijdschr Geneeskd. 132, 1081-1083 (1988).

9. Meade, T. W. et al. Epidemiological characteristics of platelet aggregability. BMJ. 290, 428-432 (1985).

10. Tschopp, T. B. \& Baumgartner, H. R. Enzymatic removal of ADP from plasma: unaltered platelet adhesion but reduced aggregation on subendothelium and collagen fibrils. Thromb Haemost. 35, 334-341 (1976).

11. Mehta, R. H. et al. Race, Bleeding, and Outcomes in STEMI Patients Treated with Fibrinolytic Therapy. Am J Med. 124, 48-57 (2011).

12. Brown, H. A. et al. Race as a predictor of morbidity, mortality, and neurologic events after carotid endarterectomy. J Vasc Surg. 57, 1325-1330 (2013).

13. Copenhaver, B. R. et al. Racial differences in microbleed prevalence in primary intracerebral hemorrhage. Neurology. 71, 1176-1182 (2008).

14. Mak, K. H. et al. Ethnic variation in adverse cardiovascular outcomes and bleeding complications in the Clopidogrel for High Atherothrombotic Risk and Ischemic Stabilization, Management, and Avoidance (CHARISMA) study. Am Heart J. 157, 658-665 (2009).

15. Desta, Z., Zhao, X., Shin, J. G. \& Flockhart, D. A. Clinical significance of the cytochrome P450 2C19 genetic polymorphism. Clin Pharmacokinet. 41, 913-958 (2002).

16. Hjemdahl, P., Larsson, P. T. \& Wallén, N. H. Effects of stress and $\beta$-blockade on platelet function. Circulation. 84, VI44-VI61 (1991).

17. Meirelles, L. R. et al. Chronic exercise leads to antiaggregant, antioxidant and antiinflammatory effects in heart failure patients. Eur J Prev Cardiol. doi: 10.1177/ 2047487313491662 (2013).

18. Wang, J. S. et al. Different effects of strenuous exercise and moderate exercise on platelet function in men. Circulation. 90, 2877-2885 (1994).

19. Naesh, O., Hindberg, I., Trap-Jensen, J. \& Lund, J. O. Post-exercise platelet activation--aggregation and release in relation to dynamic exercise. Clin Physiol. 10, 221-230 (1990).

20. Jacobs, B. S. \& Greenberg, S. M. Statins, low cholesterol, and hemorrhagic stroke: an uncertain triangle. Neurology. 70, 2355-2356 (2008).

21. Goldstein, L. B. et al. SPARCL Investigators. Hemorrhagic stroke in the Stroke Prevention by Aggressive Reduction in Cholesterol Levels study. Neurology. 70, 2364-2370 (2008). 
22. Zutt, R., van der Kooi, A. J., Linthorst, G. E., Wanders, R. J. \& de Visser, M. Rhabdomyolysis: Review of the literature. Neuromuscul Disord. 24, 651-659 (2014).

23. Kaste, M., Hernesniemi, J., Somer, H., Hillbom, M. \& Konttinen, A. Creatine kinase isoenzymes in acute brain injury. J Neurosurg. 55, 511-515 (1981).

24. Koyama, T., Shimada, M., Baba, A., Kosugi, R. \& Akaishi, M. Effects of early reperfusion on creatine kinase release in patients with acute myocardial infarction: implications for reperfusion injury. Int J Cardiol. 155, 335-7 (2012)

25. Gonzalez, E. et al. Trauma-Induced Coagulopathy: An Institution's 35 Year Perspective on Practice and Research. Scand J Surg. 103, 89-103 (2014).

26. Knudsen, J. B., Gormsen, J., Skagen, K. \& Amtorp, O. Changes in platelet functions, coagulation and fibrinolysis in uncomplicated cases of acute myocardial infarction. Thromb Haemost. 42, 1513-1522 (1980).

\section{Acknowledgments}

Prof. M. de Visser, Department of Neurology, Academic Medical Center, Amsterdam, is acknowledged for critically reading the protocol and providing useful comments. L.M. Brewster is a recipient of a VENI fellowship (grant number: 916.10.156) awarded by the Netherlands Organisation for Scientific Research (NWO) as part of its Innovational Research Incentives Scheme. The funding body had no role in the study design, nor in the collection, analysis, and interpretation of the data, the writing of the manuscript; or in the decision to submit the manuscript for publication.

\section{Author contributions}

L.B. conceived the study, and designed the experiments with D.H., R.N., K.B., M.S., G.M., J.C. and A.S. D.H., K.B. and M.S. performed the tests and data collection under supervision of R.N., G.M., J.C., A.S. and L.B. D.H. and L.B. analysed the data. All authors joined to interpret the data, and write and critically revise the manuscript. All authors gave final approval of the version to be published. L.B. guarantees the scientific integrity of the work.

\section{Additional information}

Competing financial interests: LMB is an inventor on NL patent WO/2012/138226 (filed).

How to cite this article: Horjus, D.L. et al. Creatine kinase inhibits ADP-induced platelet aggregation. Sci. Rep. 4, 6551; DOI:10.1038/srep06551 (2014).

This work is licensed under a Creative Commons Attribution-NonCommercialNoDerivs 4.0 International License. The images or other third party material in this article are included in the article's Creative Commons license, unless indicated otherwise in the credit line; if the material is not included under the Creative Commons license, users will need to obtain permission from the license holder in order to reproduce the material. To view a copy of this license, visit http:// creativecommons.org/licenses/by-nc-nd/4.0/ 